\title{
Les limites entre la religion et la culture à l'occasion de l'interprétation de la religion chinoise antique
}

\author{
Par GUNNAR SJÖHOLM
}

Dans un article intitulé "Problems for the worshipers "1 James Gordon nous montre comment, dans la Chine de nos jour, les "external signs that religious faith is being kept anywhere but in the heart " sont de plus en plus en train de disparaître. Quand un prêtre demande à une vieille réfugiée de l'interieur si elle a utilisé son chapelet pour ses prières pendant les dernières années, elle répond : "J'ai utilisé mes doigts. " Le service divin public s'est transformé en un conventicule. L'organisation religieuse est dominée par l' "Union patriotique nationale " dans l'esprit du temps. Depuis 1964 l'emprise sur le mahométisme du Sin-kiang et le bouddhisme du Tibet en particulier n'a fait que croître. Les objets du culte de la religion populaire sont pour les jeunes gardes rouges d'un accès aussi facile que les chapelets catholiques. On ne peut plus acheter d'encens ni les sortes de papier requis pour les services votifs et surtout funéraires. La divination, l'interprétation des oracles, les rites nuptiaux, les cérémonies saisonnières et le culte des ancêtres n'osent plus s'exposer à la dérision publique. Les religions recherchent l'ombre et le repliement de même qu'au sein du christianisme le quakerisme et la prière constante des philocaliens où l'un trouve aussi quelque chose à replier sur soi-même. On trouve là la conscience des arguments avancés sur le "plan culturel " avec la presse comme organe public. Le Kuang-ming Jih-pao de Pékin publia en 1965 un débat de Yu Hsiang et de C. W. Liu sur la question de savoir si la superstition féodale et la religion sont le même phénomène et si elles doivent donc être combattues

${ }^{1} C R S$, Hongkong, août 1966. 
avec les mêmes méthodes. Les auteurs constataient qu'elles ne pouvaient pas être déclarées identiques mais qu'elles avaient leur origine dans la même croyance naïve en des forces surnaturelles. La propagande athéiste devait être intensifiée pour libérer le peuple des chaînes de la superstition religieuse, et il fallait poursuivre dans relâche la lutte contre ceux qui croient en Dieu, à Bouddha ou aux génies du taoisme. Un point de vue différent fut exprimé par H. C. Ya. Selon lui toute religion est superstition, mais toute superstition n'est pas religion. Le parti doit avant tout exterminer la superstition nuisible de nature non religieuse. On reprocha à cette manière de voir d' "émbellir la religion » et d'affaiblir la lutte antireligieuse.

Dans le combat culturel de la Chine actuelle on constate un divorce entre religion et culture. L'auteur d'un article de China News Analysis, Hongkong, déc. 1965, penserait que c'est le moins qu'on puisse dire. «Religions (in China), one can say, are gone. The overshadowing of freedom of religion, which was openly proclaimed in the 1954 Constitution, is total. " Une telle affirmation est cependant en désaccord avec la dialectique de la pensée maoïste, qui dans une telle situation suggérait immédiatement l'idée d'une renaissance possible de la religion et d'une nouvelle contexture culturelle. Et il resterait par aillerus la connaissance de l'existence du phénomène religieux ailleurs, dans des sociétés avec lesquelles toute coexistence est impossible. La dialectique et le processus de la dialectique pour ainsi dire itérative ont un caractère de nécessité et d'actualité toujours renouvelée.

Les réflexions présentées jusqu'ici ont justement un trait d'actualité, dont on pourrait penser qu'il porte préjudice à l'opération theorique indiquée dans notre rubrique. Il n'en est cependant rien. Il se révèle en effet que l'interprétation de phénomènes distants dans le passé est d'autant plus dépendante de prémisses beaucoup plus rapprochées. Le degré de difficulté du problème semble demander des instruments d'une précision nouvelle et tout se passe comme si cette précision, l'analyse et la combinaison des prémisses étaient souvent presque une fin en soi, tandis qu'en fait les méthodes instrumentales devaient être adaptées aux données, aussi énigmatiques et rares qu'elles puissent être, et de préférence conçues comme tentatives. L'actualité des méthodes et les vertus que leur attribuent leurs contemporains trahit souvent la distance qui les sépare du problème posé. 
Et il n'arrange en rien les choses d'utiliser des méthodes, considérées comme nouvelles, sans rendre compte de ce qu'elles doivent à des types de pensée préexistants. Chez Mao on ne trouve aucune reddition de comptes pour ce qu'il doit à la pensée chinoise classique ni pour sa dépendance de la pensée marxiste dans sa génèse, son développement et sa structure.

Chez un des plus grands historiens de la philosophie contemporains, Fung Yu -lan ${ }^{1}$, on trouve un tel exposé de l'utilisation de méthodes de recherche occidentales traditionnelles et de leurs résultantes lors de la confrontation avec par ex. le problème de la religion et de la culture, mais l'auteur est tellement dépendant de ces méthodes qu'il s'est appauvri, sinon depouillé complètement, de ses propres possibilités, beaucoup plus grandes, de venir à bout de problèmes éminemment chinois de religion et de culture. Nous en verrons des exemples dans un instant. Il faut cependant rendre hommage à tout chercheur qui, comme Fung et son traducteur Derk Bodde, dans l'intérêt de la pédagogie scientifique, renonce à l'assurance de sa démarche dans des domaines qui lui sont familier pour extraire d'une jungle terminologique étrangère des instruments propres à faciliter l'accès des étrangers aux composantes universelles de données épistémologiques particulières. Il sera impossible de prétendre à l'universalité tant que les maigres introductions qui nous présentent par ex. la philosophie chinoise et la culture indienne au moyen de raccourcis pédagogiques et dans une incompréhensibilité inimaginable continueront à ne même pas poser la question d'éventuels dénominateurs communs et à laisser maner creux des termes changés pour un Occidental de connotations fausses. Il ne devait par ex. pas être permis de présenter, sous la clé de la religion, la « nuit du moyen âge » européen sans donner en même temps, sous la clé de la culture, une peinture du « soleil de la dynastie Tang»en Extrême-Orient, ni d'ignorer l'équation culturelle et religieuse contenue dans le millésime izoo ou la contemporanéité de Saint Thomas et de Chu Hsi.

La pensée dialectique qui, avec la pensée existentialiste et la pensée logistique, et au plus grand dam de la pensée critique, caractérise notre époque n'a pas toujours nécessairement affirmé l'incompatibilité des valeurs religieuses et culturelles. Hegel a forligné chez des penseurs qui, avec des

1 Voir $A$ History of Chinese Philosophy. Peiping 1937. 
méthodes inverses, sont arrivés à des résultats diamétralement opposés, encore que, chez ceux-ci, la religion, traitée au début comme un épiphénomène tendant à la valeur zéro avant de devenir une valeur négative, subitement et "ironiquement " devient un phénomène qui, sous les habits d'une "idéologie " prend le rôle d'une valeur prime conditionnant les formations de blocs dans le procès d'évolution de la société. La philosophie de la religion de Hegel - qui en soi représente une valeur culturelle - nous intéresse dans notre contexte non seulement parce qu'elle forme la base de sa pensée en général, mais encore parce qu'elle comporte une manière d'interpréter les religions antiques, entre autres la religion chinoise, en même temps qu'elle implique une théorie générale des religions valable aussi pour l'analyse de l'évolution culturelle de l'humanité. La tradition hégélienne peut être suivi dans un habile résumé de Torsten Bohlin, Kierkegaards dogmatiska åskådning $i$ dess historiska sammanhang1. (Je traduis :) «Tandis que la philosophie, par suite d'une nécessité interne a comme but final la connaissance fondamentale, la philosophie de la religion est nécessairement liée au système général de la philosophie et en marque le point culminant. $»^{2}$ Une unité de principe de la croyance et de la connaissance caractérise la raison humaine et " en vertu de ce principe il est exclu que le contenu de la religion positive, à condition d'être bien compris, soit en contradiction avec la raison humaine ". Hegel distingue trois composantes dans la "notion de religion ": l'idée de Dieu, la conscience de Dieu et le culte. Sous sa forme la plus simple le culte est recueillement ou union mystique, la présence de Dieu dans l'homme ou le fait, chez Dieu, de "devenir homme ». Vue sous cet angle, la "notion de religion » est la recherche religieuse de la vérité, qui se réalise sous forme de procès dans les différentes religions et dans l'histoire universelle. Les différentes religions ne sont pas coordonnées mais représentent une évolution. Au premier stade, celui des religions naturelles, Dieu est conçu comme une unité naturelle entre l'esprit et la nature, comme une puissance naturelle absolue par rapport à laquelle l'homme ne ressent aucune liberté. Au nombre des religions naturelles Hegel compte la religion chinoise, "religion de la mesure ", la religion indo-

\footnotetext{
${ }^{1}$ Kierkegaards dogmatiska åskådning $i$ dess historiska sammanhang (La conception dogmatique de Kierkegaard dans son contexte historique), chap. 7, I925.

${ }^{2}$ Hegel, Werke, XI, I832, p. 3 ss.

$8-68438$ I Hartman
} 
brahmanique, "religion de l'imagination " et le bouddhisme, "religion de l'être en soi ». Au deuxième stade l'idée de Dieu se trouve à un point de transition entre substantialité et objectivité et distinct de l'individualité humaine. Les religions transitoires seraient les religions perse et égyptienne, du bien (de la lumière) et du mystère qui se rapprochent de l' "individualité spirituelle \%. L'individualité est encore plus accusée au troisième stade, caracterisé par la "subjectivité libre et déterminée 》 dont l'activité consiste en une action appropriée à un but. Là se situent les religions juive, grecque et romaine, "religions du sublime, de la beauté et de la rationalité ", la religion romaine se trouvant à la limite de la religion absolue, qui est le christianisme dans lequel le contenu de la notion de religion est devenu objet de la conscience religieuse. Puisque la notion de religion a été essentiellement définie comme l'autoconscience de Dieu, ceci signifie que la conscience finie qui possède ce contenu est elle-même un stade de ce procès qui a lieu au sein de Dieu. La définition donnée par Hegel de la religion chinoise comme une "religion de la mesure 》 donne lieu à une remarque critique de première importance : que penser d'une méthode dialectique qui fait de l'absolu le point terminal d'un développement d'idées déterminé par une idée de développement traversant une série de stades caractérisés par un degré relativement élevé d'absolu?! C'est à cette démarche alogique que s'attaque entre autres la critique existentielle de Kierkegaard. Funeste pour l'interprétation de rapport entre la foi et le savoir, la religion et la culture dans l'esprit hégélien est l'argumentation dialectique qui avec la même méthode mais d'une manière diamétralement opposée définit la valeur "culturelle " fondamentale comme relevant essientellement du domaine " naturel ", "materiel " ou "économique ». La terminologie est identique, mais la vue globale des choses est inversée. On peut étudier cette situation paradoxale dans le passage suivant de Mao Tse-tung ${ }^{1}$ : A Marxist recognises that the development of the total process of the universe is absolute, whereas the development of each particular process in this total process is relative. Hence in the great river of absolute truth man's knowledge of a particular process in each given stage of development is only relatively true. Absolute truth is compounded of a sum-total of relative truths. "Il vaut aussi la peine

1 Mao Tse-tung, On practice, Pékin 195I, p. 21. 
de citer un autre passage. S'il se peut que le texte que nous venons de voir dévoile un rapport inattendu entre une démarche hégélienne et - mutatis mutandis - un mode de pensée marxiste moderne devant un exemple de diffusion de phénomène culturel qu'il faudrait étudier de plus près, la citation suivante peut dévoiler une perspective plus large et ouvrir les yeux sur un type de structure de la pensée dialectique déja illustré dans la pensée chinoise classique ${ }^{1}$ et, strictement parlant, aussi dans la pensée occidentale et hellénistique d'inspiration plus ou moins orientale. Le terme maoiste de «practice » doit ici être compris à la lumière de la définition hégélienne du culte comme l'expression, non seulement d'un savoir, mais aussi d'une forme particulièr d'action, au moyen desquelles on parvient à une certitude religieuse, à une vérification obtenue par l'expérience au sens large du mot. "The discovery of truths through practice and through practice the verification and development of them; the active development of perceptual knowledge into rational knowledge and by means of rational knowledge, the active direction of revolutionary practice and the reconstruction of the subjective and the external world; practice, knowledge, more practice, more knowledge, and the repetition and infinitum of this cyclic pattern and with each cycle, the elevation of the content of practice and knowledge to a higher level : such is the whole epistemology of dialectal materialism, such is its theory of the unity of knowledge and action $\eta^{2}$. Le modèle structural de la pensée dialectique peut être élaboré en patron théorique valable pour toute espèce de domaine d'expérience religieux ou culturel, en général, pourvu que ce domaine soit assez vaste pour que ses traits distinctifs puissent être considérés comme possédant un contenu global, un thème fondamental. "L'appareil conceptuel de l'hégélianisme est comme on sait conservé aussi bien dans le marxisme que dans l'existentialisme ${ }^{3}$.

Le philosophe Hegel a donc trouvé que l'évolution de la religion et de la culture se conditionnent réciproquement et que, parmi d'autres types de religion, la religion chinoise se rattache à la "mesure " comme une valeur primaire de nature religieuse. Le philosophe de la société Mao considère la religion dans le contexte culturel comme un phénomène passager indési-

1 Fung, A History, chap. XI, p. 258, The later Mohist school.

2 On Practice, p. 23, Conclusion.

3 Anders Wedberg, Filosofins historia, III, p. I5. 
rable, une "superstition féodale " sans attache dans la réalité. Fung Yulang, qui a ses racines dans le monde classique, ne peut pas s'empêcher de constater que la religion est un phénomène primaire, mais dans son désir d'inscrire la pensée chinoise dans un cadre mondial il suit certains modèles occidentaux et déclare constater un désattachement croissant et une libération de la pensée par rapport aux conceptions religieuses. Il semble osciller entre une position comme celle de Spengler : «Kein Glaube hat je die Welt verändert 》; de Marx : "... die Religion selbst samt der übrigen Ideologie auf die wirtschaftlichen Vorgänge zurückzuführen sind »; de Comte : "dass der wirkliche Fortschritt der Kultur sich gerade in der Befreiung von der Religion vollzieht 》 et celle de E. Fueter et J. Haller selon laquelle l'évolution politique et culturelle est caractérisée par une «strenge Eigengesetzlichkeit " par rapport à l'évolution morale et religieuse'. Une fois opérée cette entaille sécularisante, Fung n'a aucun mal à faire entrer Aristote dans Confucius et le confucianisme ${ }^{2}$, Platon dans Mencius ${ }^{3}$, Descartes dans Chuangtsé4, Hobbes et Bentham dans Meh tsé5, William James dans Confucius et Hsuen tsé́ et les sophistes dans le méhisme tardi. Fung termine par example de la façon suivante un résumé de données historiques se rapportant à l'interprétation du sacrifice selon le $\mathrm{Li}$ chir : «This is identical with what Comte calls the 'religion of humanity'. It is based on sacrifices performed to express gratitude to men who have done great deeds in the past ... Heaven and Earth, stars, constellations, birds, beasts, trees, etc. are likewise sacrified to out of feelings of gratitude. This practice, while it may have originated from animism, has, because of the interpretation given it by the Confucian school, become poetical rather than religious. $1^{8} \mathrm{La}$ déthéologisation et la psychologisation des rites sacrificatoires permettent à Fung de transposer la valeur religieuse en une valeur esthétique, dont le fondement théorique peut à la rigueur être considéré comme philosophi-

1 Cf. Karl Holl, Gesammelte Aufsätze. p. chap. 8, 1923.

3 Fung, op. cit., pp. 122, 37 I.

3 Op. cit., p. 117 .

4 Op. cit., p. 227.

5 Op. cit., pp. ror, ro3, 96, 249.

6 Op. cit., pp. 239, $28 \mathrm{r}$.

7 Livre des rites, compilation confucianiste du $I I I^{e}$ s. avant $\mathcal{Y}$.-C., chap. 46.

8 Fung, op. cit., p. 354 . 
quement moins compromis que celui de la religion. Religion et théologie reçoivent leur coup de chapeau en tant qu'activités « innerhalb Grenzen der praktischen Vernunft " et que structuralisation parmi d'autres, de l'esthétiquement valable, de ce que Kant appelait " ein uninteressiertes Wohlgefühl ». Tel est, sur le marché philosophique, le prix de la carte de membre de la Société de la Raison Vitale. En fin de compte c'est une question encore ouverte que de savoir si Fung considère qu'avant Confucius (55I479 avant J.-C.) il a existé quelque forme de pensée systématique. Cependant son troisième chapitre porte le title : "Pensée philosophique et religieuse avant Confucius. "Toutefois, dans ce chapitre, il semble inclure Confucius lui-même et son temps - peut-être parce que Confucius a déclaré : "Je m'en tiens à Chou " (la dynastie bien connue I I22?-256)1. Avec une espèce de zèle apologétique Fung développe une idée sur le contenu de la notion de pensée philosophique systémaqtique. Il affirme qu'en réalité la philosophie chinoise a des qualités systématiques de classe aussi élevée que la philosophie occidentale même si celle-ci est supérieure dans le domaine de la forme. Par contre Fung ne dit rien sur la question de savoir si la pensée religieuse de la Chine classique a des qualités formelles et réelles - possède logique externe et interne. L'étalon serait la rationalité. Mais toute la pensée philosophique chinoise n'est pas rationnelle, ni au sens formel ni au sens réel du mot. La distinction opérée par Fung qui caractérise la civilisation préconfucianiste comme une civilisation religieuse et considère pratiquement le scepticisme et la critique rationaliste de la religion comme la condition d'une pensée pure digne de nom de philosophie ne trouve guère d'appui dans les textes sur lesquels il fonde cette opinion. Même si elle était juste, l'ironie - ce correctif omniprésent - serait là pour nous rappeler un état de fait formulé par Fung lui-même de la façon suivante : "Suffice it to say that (about the time of the birth of Christ) the divinity of Confucius ... has been one of the points of contention (between the Old and the New Text-school) and that in this respect, at least, the Old Text-school has been more rational and less superstitious than the New Text-school. $\|^{2}$ Il est parfois risqué de faire des distinctions et de louer la fraîcheur de l'ombre sans dire du bien de 1'arbre. En Chine cela s'appelle "shu lin hsia », dans l'Inde

1 Cf. Lun $Y u$, III: I4.

2 Fung, op. cit., p. I8, note I. 
l'arbre de Bodhi, dans le Nouveau Testament "ficus religiosus". "Ngo tsai wu-fa-ko-shu ti hsia, gno chiu kan chien ngi liao » - « quand tu étais sous le figuier, je t'ai vu » (Jean $I, 48$ ).

Dans son chapitre 2 Fung, s'appuyant sur des citations choisies, nous décrit la civilisation chinoise sous la dynastie Chou comme parvenant à une structure véritable grâce à une exclusion sans cesse croissante de la religion. Selon sa manière de nous présenter les choses, Confucius marque le début d'un période de l'histoire de la pensée qu'il appelle celle des «philosophes 》 et qui aurait duré jusqu'à l'avènement de la dynastie Han (206 av. J.-C.-220 apr. J.-C.) Fung attache à ce propos beaucoup d'importance au fait que la "philosophy, if it is to be the systematic manifestation of thought, must necessarily find expression in the writings of private individuals $\%$. Avant Confucius il n'y aurait pas eu de telle activité littéraire privée mais seulement des écrits de fonctionnaires. Fung cite l'historien H. C. Chang (I738-I801) à l'appui de cette distinction entre activité litteraire privée et officielle. Mais qu'est-ce qui empêche une production littéraire officielle d'avoir des qualités systématiques? Comme par ailleurs il est douteux que Confucius a écrit lui-même les textes qu'on lui attribue, il est impossible de faire de lui le premier écrivain privé. Pour cette raison Fung insiste sur le fait que Confucius serait le premier qui, pendant une certaine période de sa vie, se serait uniquement adonné à l'élaboration et à l'explication de ses maximes de sagesse. Cette affirmation est difficile à prouver et Fung se tire d'affaire en disant que s'il est vrai que tout ce que les générations ultérieurs ont attribué à Confucius ne saurait être historique, cette tradition ne saurait cependant être complètement fausse. Au lieu de rejeter la possibilité que la civilisation chinoise ait toujours produit des manifestations pourvues d'une manière ou d'une autre de contenu systématique et donc susceptibles d'organisation et ainsi d'écarter à priori la possibilité d'une logique interne de la religion chinoise, Fung a voulu server la question de plus près et semble avoir pu trouvé, déjà dans la difficulté quasi-insurmontable de fixer la place systématique de la pensée chinoise, une erreur fondamentale qui se rattache à la supposition gratuite que la religion et la culture ne se comportent pas comme l'arbre et l'ombre mais comme le feu et Phénix ou le sable volant et le chemin tracé.

Fung base donc sa peinture de la religion et de la pensée avant Confucius 
sur la supposition d'une littérature officielle, non-individuelle, dont il est à priori entendu qu'elle ne contient rien qui mérite le noble nom de philosophie mais seulement des opinions sur des notions religieuses et la description de comportements qui supposent la croyance en des entités comme certaines divinités, la divination, la magie, le Ciel et Dieu. Au moyen d'une citation de "Dialogue des Etats 》 (Kuo Yü, env. 400 av. J.-C.) Fung démonstre " in a general way the forms of superstition of the early Chinese ». Les nombreux esprits étaient conçus anthropomorphiquement et un polythéisme général régna aussi par la suite, sous les dynasties Hsia et Shang, bien que « the concept of Heaven (T'ien) and God (Ti) arose, a monotheistic belief which seems gradually to have gained influence $»^{1}$. Fung affirme en même temps que cette religion concordait exactement avec celle des Grecs. Il constate aussi dans les "Contes de Tso » (Tso Chuan, env. V॰ s.) une coexistence de la croyance aux esprits et du monothéisme, avec dominance de la première. Cf. Tso Chuan, an $706:$ "What is meant by morality (on the part of a ruler) is to show loyality toward the people and sincerity toward the spirits. When the ruler thinks of benefiting the poeple, that is loyalty. When the priest is truthful in his words, that is sincerity. " Mais, selon "Kuo Yü ", le "Maître Superème ", Shang Ti, n'est pas lui-même rangé parmi les nombreuses divinités. Fung nous fait en réalité entrevoir un croyance qui n'est pas souillée par toute sorte de superstitions, mais il la passe ensuite sous silence dans son exposé du développement ultérieur de la pensée. Pour exclure le plus possible la superstition de l'histoire de ce développement, Fung fait appel aux sentences débattues de Confucius : "One should respect the spirits, but keep them at a distance " (Lun Yu, $\mathrm{VI}, 20$ ) et "He sacrificed to his ancestors as if they were present " (ibid., III, I2). Ces deux sentences témoigneraient selon Fung d'une attitude sceptique. La seconde ne doit cependant pas être citée sans son contexte, à savoir, selon la traduction de Wingtsit Chan" ${ }^{2}:$ "When ... he offered, he felt as if his ancestral spirits were actually present ... He said, 'if I do not participate in the sacrifice, it is as if I did not sacrifice at all'. " Pour ce qui est de la première, Wing en donne le commentaire suivant : "The passage means either 'do not become improperly informal with spiritual being'

1 Fung p. 24.

${ }_{2} A$ Source Book in Chinese Philosophy, p. 25. Princeton 1963. 
or 'emphasize the way of man rather than the way of spirits'. " Bernhard Karlgren ${ }^{1}$ n'y voit pas non plus d'expression d'agnosticisme (je traduis du suédois) : "Dans cette sentence Confucius n'a pas en réalité voulu faire preuve de négativisme ou de scepticisme; il a seulement voulu dire qu'il ne fallait pas être familier, indiscret envers les Esprits. " En ce qui concerne le chapitre III: I de Fung, il ne reste qu'à constater une incertitude de méthode dans l'exposé, même s'il semble avoir été influencé par une conception évolutioniste des phénomènes religieux plus courante autrefois qu'aujourd'hui. La première phrase de sa première citation lui crée déjà des difficultés : "In ancient times people and divine beings did not intermingle. »² Mais Fung ne s'arrête pas à cette phrase et utilise seulement les passages de la citation qui donnent une meilleure assise à une théorie évolutioniste. Il souligne la coexistence du monothéisme et du polythéisme mais sans analyser aucun. Considère-t-il qu'une conception monothéiste est compatible avec une pensée avancée? Il laisse la question entière.

La superstition préphilosophique est traitée dans le chap. III: 2 sous la forme d'un résumé d'un exposé sur la divination et la magie (Pan $\mathrm{Ku}$, 32-92 apr. J.-C., où il est fait utilisation d'une documentation réunie par Liu Hsiang, $77-76$ av. J.-C., et Liu Hsin, env. 53 av.-23 apr. J.-C.). Il y a six classes : l'astrologie, l'almanachologie, les cinq éléments, la divination au moyen de l'achillée (shi) et des carapaces de tortue (pu), l'oniromancie, l'interprétation des chiffres, des formes, etc.

Dans le chap. III: 3 (avec citation des sources déjà mentionnées ainsi que des "Hauts Documents ", Shu Ching, avec leurs matériaux des $\mathrm{X}^{\mathrm{e}}$ et $\mathrm{IX}^{\mathrm{e}}$ s. av. J.-C., et des "Odes ", Shi ching, poèmes des $\mathrm{X}^{\mathrm{e}}-\mathrm{VII}{ }^{\mathrm{e}}$ s.) Fung donne un aperçu des notions de Ciel (T'ien) et de Dieu (Ti), représentant une image physique, anthropomorphe, fataliste, naturaliste ou éthique. Fung se dispense de plus de commentaires et passe sans transition au chap. III: 4, intitulé Beginnings of enlightenment, et trouve dans les sources que nous venons de nommer des exemples de penseurs qui progressivement perdent la croyance en la surnaturalité des divinités et en la "voie du Ciel " (tao). Ce scepticisme se rattache aux idées de yin et de yang, ces deux principes naturels qui expliquent suffisamment les phénomènes de l'univers.

1 Bernhard Karlgren. Religion i Kina, Antiken, p. 87. Stockholm I964.

2 Propos de Kuan I Fu, env. 500, Ch'u Yü, II, I. 
La notion du Ciel se trouve influencée par ce type de pensée qui à l'époque en question fut marqué par le naturalisme taoiste. Cette période débouche selon Fung sur The rise of rationalism (chap. III: 5). Celui-ci se traduit moins en des réflexions autour de l'essence et de l'origine de l'univers qu'en une explication de l'organisation de la société et de l'origine des institutions humaines, des lois régissant la vie politique et sociale. Fung cite les «Odes》: "Heaven gave birth to the multitudes of people, so that they had faculties and laws ", "without consciousness, without knowing, be in accordance with the pattern of God " (Odes III, iii, 6: I et III, i, 7: 7). Il s'agit donc d'un modèle divin sur lequel régler l'organisation de la société (tseh). Dans les "Kuo Yü 》 on trouve cependant des déclarations selon lesquelles les lois auraient été instituées par les hommes et pour les hommes. On en vint à considérer même les rites officiels comme des "prétextes", des moyens en vue de créer unité et discipline dans la société. Lorsque le doit divin n'est plus en vigueur, on est amené à reconnaitre le bien fondé du régicide. Fung trouve dans les textes classiques des parallèles à la sentence de Protagoras que l'homme est la mesure de tout. Ensuite il ménage la transition à son propre exposé de l'histoire de la philosophie chinoise (" more important contributions to Chinese thought») en rappelant le fait que les auterus des textes classiques, préphilosophiques, étaient tous détenteurs de charges héréditaires aux cours ou de toute façon des personnes que leurs situations officielles empêchaient de s'exprimer librement. De tels empêchements n'auraient pas été présents pendant la "period of Philosophers \%. En fait Fung se révèle lui-même comme empêché par diverses circonstances d'appliquer à son propos un point due vue systématique qui aurait donné à l'exposé des rapports entre la religion et la culture l'unité dont il manque. Wingtsit Chan ${ }^{1}$ cite un passage de Confucius qui pourrait se rapporter à tout l'ouvrage de Fung: "To go too far is the same as not to go far enough. » Celui qui dit que la religion n'est que superstition "va trop loin »; mais il ne va pas non plus "assez loin ", car la croyance n'implique pas seulement la croyance elle-même mais tout ce qui s'y rattache, et tout ce qui s'y rattache n'est pas superstition mais l'ensemble de la culture, tout ce qui est jugé comme bon.

1 Chang, A source Book ..., p. 37. 
Quel aspect prend maintenant la question de la religion et de la culture aux yeux de la recherche qui fait usage des méthodes de la linguistique et de la critique textuelle? Le chercheur doit en premier lieu avoir à sa disposition les textes les plus sûrs possibles. Il doit aussi se rappeler que de nouvelles découvertes fournissent sans cesse des matériaux nouveaux qui parfois contraignent à des retouches importantes de l'image qu'il s'est faite du passé. Pour ce qui est de la Chine il vaut la peine de remarquer que sa culture à l'époque du passage de l'époque préhistorique à l'époque historique - marquée par la présence de documents littéraires — est déjà une structure solidement constituée. Fung renvoie à ce sujet aux textes courants. Il faut cependant tenir compte de la découverte à An-yang en 1899 de milliers de carapaces de tortue portant des inscriptions en écriture chinoise archaïque. Les bronzes (du $\mathrm{I}^{\mathrm{er}}$ millénaire) étaient déjà connus et considérés depuis longtemps comme d'une étude difficile. Les carapaces de tortue étaient un sujet encore plus difficile. Vinrent s'y ajouter au cours des années 20 et suivantes de nouvelles découvertes, partiellement du même ordre, témoignant d'une culture élevée de l'âge du bronze. Les textes divinatoires ont été mis à la portée du public suédois pour la première fois par Bernhard Karlgren dans son ouvrage "Religion i Kina, Antiken"1. Précédemment ces textes avaient été transcrits en écriture chinoise normale par Ch'en Meng-chia. Mais celui-ci ne les avait ni commentés ni traduits en chinois moderne. La contribution de Karlgren est donc d'autant plus précieuse. Importante pour l'interprétation est aussi sa remarque que la population de l'aire culturelle Shang-Yin n'était pas homogène et que des composants émanant des tribus montagnardes aux langues et aux mœurs différentes ont dû marquer cette culture. De plus ces matériaux ne donnent qu'une image fragmentaire de la religion de l'époque, étant donné que les croyances et les usages du commun restent dans l'ombre. Ils nous renseignent seulement sur ce qui avait cours chez la noblesse et la famille royale. L'image fournie par ces textes est corroborée par les vases sacrificatories de bronze trouvés dans des tombeaux et portant parfois des inscriptions comptant jusqu'à 40 mots. Ces inscriptions confirment l'existence d'un culte des ancêtres qui est aussi une des plus courantes révélations des textes divi-

${ }^{1}$ Religion $i$ Kina, Antiken, pp. 19-26. 
antoires. Ceux-ci ont plus d'une trentaine de termes pour la notion de sacrifice aux esprits, à qui on fait des offrandes qu'ils sont chargés de transmettre à la divinité. Il est fait une distinction entre les esprits naturels (shen) et les âmes ou esprits des morts (kuei); certes non sans labilité, puisque, parmi la vingtaine d'ancêtres de T'ang, le premier en ordre des 36 rois Shang-Yin, on trouve T'u, l'esprit du sol, et Ho, l'esprit du Fleuve Jaune. Il est parfois donné des exemples de souverains et d'hommes d'état dont l'âme a été promue au rang d'esprit naturel. A l'origine on ne discerne aucun lien de sang entre le "Maître Suprème " (Shang Ti ou Ti), puissance qui régit sous les autres esprits naturels, et les ancêtres. Shang Ti règne sur le vent, la pluie, le temps, les fruits de la terre et donne aux souverains grâce, disgrâce, approbation, disapprobation et admonitions. Les sacrifices ne sont adressés directement à Shang Ti qu'en tant que sacrifices d'hommage. Par ailleurs, et en général, ils sont adressés à l'esprit des ancêtres et celui-ci transmet les prières au Maître Suprème. Ces sacrifices sont rituellement célébrés par le roi lui-même, assisté d'un oracle. Les sacrifices sur les autels de plein air sont souvent célébrés par les chamanes (hi 'sorcier' et wu 'sorcière'), rompus aux danses extatiques d'invocation des esprits. Karlgren, dont nous venons à grand pas de suivre l'exposé, exerce dans son chapitre II, sur la croyance et le culte sous la royauté et la féodalité, une critique serrée des sources classiques, depuis les «Hauts documents 》 jusqu'à Meh tsé, telles qu'elles ont été copiées et transmises oralement jusqu'à leur fixation plus ou moins définitive sous la dynastie des Han (206 av.-220 apr. J.-C.). Pour son propre compte Karlgren base son exposé sur les textes divinatoires et autres que nous avons mentionnés précédemment et sur ces "sources libres », non sur les versions canoniques. Il exclut ainsi des sources comme Yi Li, Chou Li et Li Chi (Manuel Rituel, Chronique des Chou, Mémorial des Rites) en tant que systématisations tardives, reconstructions, en particulier pour ce qui touche à l'histoire de la religion. Malheureusement les auteurs occidentaux ont souvent appuyé leurs études de la religion de l'époque Chou (Io27-256 av. J.-C.) sur ce "fatras de spéculations reconstructives ». Une forme encore plus risquée de recherche historique est celle qui utilise comme source la poésie mythologique du $I^{e} s$. (Ch'u ts'i), consignée dans le "Livre des Montagnes et des Mers" (Shan Hai Ching), qui est un inventaire des idées folkloriques contemporaines d'une Chine 
qui avait déjà colonisé le Sud et incorporé des tribus de cultures, de religions et de folklores différents, de même que, par la voie de l'Asie Centrale, elle était entrée en contact avec le Proche Orient. Quand de tels phénomènes postféodaux sont supposés valoir pour l'époque féodale aussi, les résultats obtenus sont souvent loin de la réalité. Karlgren analyse en particulier les sources de l'époque Chou sur l'âme humaine, composée de P'o, l'âme animale (spermatique), et de Hun, l'âme intellectuelle. Ce qui captive le plus est cependant son interprétation du terme T'ien, le Ciel, terme que l'on ne rencontre jamais dans les textes divinatoires de l'époque Shang-Yin mais bien dans ceux de l'époque Chou. $\mathrm{I} l$ ressort que Karlgren, qui a précisément décrit Shang Ti comme une force de la nature, considère qu'il a été conçu comme une divinité personnelle, le Maître Suprème. Et ceci vaut aussi pour T'ien, qu'il faut concevoir comme un synonyme de Shang Ti, par exemple dans l'expression Huang T'ien Shang Ti (Le Maître Suprème du Ciel Majestueux). T'ien est écrit sur les plus anciens bronzes au moyen d'un signe très nettement anthropomorphique. De plus le Ciel ou (Shang) Ti est maintenant associé à la dynastie Chou, ce qui n'avait pas eu lieu précédemment. "La première mère de celle-ci avait posé le pied sur l'empreinte du gros orteil du Maître ce qui l'avait 'égayée et bénie' (=fécondée)" (Ode 45). Dans les plus anciens documents Chou le roi est appelé T’ien Tsi, le Fils du Ciel.

Karlgren nous donne ensuite, selon les "sources libres 》 un exposé du culte des esprits naturels et en particulier de la divination qui survécut pendant toute la période. La technique des oracles est maintenant complétée par l'usage de l'achillée (Shi) et une méthode particulière pour interpréter les réponses reçues au moyen de cette manipulation. Notre source de connaissance est ici Yi Ching (Livre des Transformations), dont la partie principale est au nombre des premiers textes Chou. (Soit dit en passant : se méfier des spéculations - telles que celles de Maspéro - sur Ming-t’ang, texte Chou tardif.) Karlgren termine ce chapitre avec une présentation des officiants adjoints du roi et des dignitaires féodaux : oracles techniques ( $p u-j e n)$, intreprétations (chan-jen), oniromanciens (chang-meng), grand-prêtre du temple des ancêtres (tsung), une série de prêtres priants $(c h u)$, chamanes ( $h i$ et $w u$ ). Rapportons enfin un point de vue appliqué par Karlgren dans son troisième chapitre (Confucianisme et moïsme) et qui met en relief la 
manière divergente dont Fung traite un problème particulièrement important. Karlgren lui aussi a conscience de l'évolution de la pensée et de la conception de la vie qui caractérise les penseurs chinois de la période Chou décadente. Mais l'introduction de la spéculation yin-yang ne signifie pas pour lui une rationalisation mais un élargissement du cadre des idées qu'on se faisait sur les missions sacrales du souverain féodal, au moyen desquelles était maintenu l'ordre sacré de l'univers. Transportée sur les notions de yin et de yang, la réflexion sur la nature de l'univers continuait à faire partie de la vie religieuse, en ce sens que "l'humanité n'était en rien située en dehors de ce système de forces de la nature mais en formait une partie intégrante ». "Il est de toute première importance pour la compréhension de l'histoire religieuse de la Chine de reconnaître justement ce fait que, même sous des formes sociales plus avancées les conceptions religieuses ont longtemps gardé beaucoup de leur naïvité primitive ... Certes on continuait à observer formellement le système rituel compliqué, mais il était devenu une routine vide de contenu. Une inquiétude générale saisit les esprits, les temps étaient mûrs pour quelque chose de nouveau. " C'est sous cet éclairage qu'il faut, selon Karlgren, voir l'action des philosophes, à savoir comme une "plantation nouvelle » sur les terres de la culture et de la religion. Les nouvelles plantes furent le quiétisme taoiste et la piété monothéiste de Confucius et de Meh tsé. Rien de tout cela ne comporta cependant de rupture avec la religion.

Signalons enfin deux points de vue, importants à la fois en roi comme sujets d'étude, et pour le traitement des problèmes que nous venons d'évoquer.

$\mathbf{I}^{\circ} \mathrm{La}$ culture chinoise nous parle depuis les origines au moyen d'une écriture qui note un vocabulaire où domine une terminologie religieuse ressortissant à la sphère de l'oracle, de la prière et du rite. Comme ici une distinction entre phénomènes culturels et religieux paraît impossible, il faut en tirer les conséquences méthodiques par l'étude de toute la religion chinoise antique. Ce vocabulaire et ces caractéristiques graphiques se développe comme un champ sémantique, susceptible d'organisation et d'examen spéculatif. Il est relié en arrière à une tradition orale, de nos jours inaccessible, portant sur l'interprétation de certains modèles (patterns) tels que certaines positions astrologiques, les phénomènes naturels insolides, 
certaines répétitions de phénomènes, certains objets de forme étrange, modèles qui lorsqu'on les reproduisait graphiquement représentaient aussi l'interprétation de ces formes; et il est relié en avant à toute l'histoire de l'écriture chinoise ultérieure avec l'évolution de la forme des caractère, leur intreprétation et leur adaption àvant tout à l'expression de nouveaux événements et de nouvelles découvertes (choses et relations humaines, sii). Sans cesse le "poids" des mots, des signes, augmente. Plus le tracé évolue, avec, entre les formes primitives et les formes "calligraphiques", souvent deux bonnes dizaines de formes intermédiaires échelonnées sur autant ou encore plus de siècles, plus chargé se fait le contenu sémantique de chaque signe. Au cours de ce procès la pensée chinoise est allée son chemin sans jamais perdre contact avec ce répertoire de signes divinatoires et d'intreprétations. Ce contact est si manifeste qu'il est superflu de le spécifier ailleurs que dans des études techniquement linguistiques. Cette richesse de connotations est en fait si grande que la langue chinoise possède un mot pour chaque type de représentation qui ait jamais traversé l'esprit d'un non-Chinois. Les difficultés de traduction et de communication ne résident pas seulement dans les mots eux-mêmes mais au moins autant dans leur contexte.

$2^{\circ}$ L'existence même de textes divinatoires chinois tels qu'ils ont été décrits par M. C. Ch'en et Bernhard Karlgren entre autres devrait fournir, à plusieurs disciplines sinologiques, l'occasion de reprendre le problème du sens de la consultation des oracles et de la magie qui s'y rattache. Autant qu'on les connaisse, les rites chinois de divination étaient exécutés en étroit connexion avec le culte des ancêtres. Ils font donc partie des expressions de la vie religieuse. Ils supposent la perplexité de l'homme et son besoin de demander conseil à la divinité. Celui qui, dans une situation donnée $(\tau \grave{\alpha}$ $x \alpha \vartheta \cdot \dot{\gamma} x o v \tau \alpha)$, ne sait pas quelle décision prendre cherche le mot qui l'aidera. Cf. l'étymologie de mot oracle : orare "parler, prier ". La littérature historique de la Chine commence par une description de la manière dont les conseils divins étaient donnés et suivis. La littérature philosophique aussi a un caractère analogue : les penseurs donnent des conseils dans une situation globale nouvelle. L'important n'est pas là de savoir si le maître est "Inner sage and Outer king " ou s'il joue le rôle non-officiel de simple "Inner

${ }^{1}$ Fung, op. cit., pp. 2, 3 . 
sage ». Souvent c'est par ses élèves qu'il parle aux dirigeants de son temps. S'il ne lui est pas libre de parler, il cultive son " teh ", sa vertu, et est convaincu que ses intentions agissent comme un puissant conseil, pour ne pas dire comme le vent qui couche les tigès de blé, que ce conseil soit suivi immédiatement ou non. Les sentences des maîtres de sagesse fonctionnent à leur époque de la même manière que les textes divinatoires codifiés ( $\mathrm{Y}$ ching), et il est aussi sûr que ces oracles étaient à l'époque accompagnés du culte des ancêtres, qui transmettaient les requêtes à Shang Ti. Le milieu est toujours celui deu rite et l'attitude des participants correspond au sens de celui-ci. L'activité philosophique qui sous sa forme extérieure représente l'idéal mystique détourné du monde ou se manifeste comme une critique des formalismes de la vie sociale ne perd dans aucune de ses démarches le contact avec le vocabulaire semé sur les carapaces de tortue et codifié dans de solides ouvrages classiques qui, dans des phrases d'un style achevé, nous font part d'une expérience multiséculaire de remèdes portés à la perplexité humaine dans des situations omineuses et de punitions souffertes par ceux qui n'ont pas obéi aux directives des signes. C'est l'écriture sémantiquement transparente qui relie les époques différentes. Religion et culture? - Dans le contexte divinatoire les deux notions se con-fondaient. Dans d'autres contextes ont été écartelées. On se rapprochera de la vérité si l'on se réfère aux traits les plus anciens de la mentalitè chinoise et reconnaît que les deux notions sont "sémantiquement proches 》. En élargissant suffisamment son champ de vision, on aperçoit cette proximité des notions. On peut appliquer à l'étude de la religion et de la culture ces mots de F. B. Fitch au sujet de la philosophie (je traduis) : "S'il est vrai, comme le dit Aristotle, que la philosophie commence avec l'étonnement et la curiosité, il est aussi vrai, qu'elle finit de même. La complexité du monde et de l'homme est en effet si grande qu'il restera toujours des mystères insondables malgré tous les progrès de la recherche et de la réflexion. „) ${ }^{1}$

\footnotetext{
1 Anders Wedberg, op. cit., p. 7.
} 